\title{
BMJ Open Changes in alcohol consumption after treatment for depression: a secondary analysis of the Swedish randomised controlled study REGASSA
}

\author{
Catharina Strid, ${ }^{1}$ Mats Hallgren, ${ }^{2}$ Yvonne Forsell, ${ }^{2}$ Martin Kraepelien, ${ }^{3}$ \\ Agneta Öjehagen ${ }^{4}$
}

To cite: Strid C, Hallgren M, Forsell Y, et al. Changes in alcohol consumption after treatment for depression: a secondary analysis of the Swedish randomised controlled study REGASSA. BMJ Open 2019;9:e028236. doi:10.1136/ bmjopen-2018-028236

- Prepublication history for this paper is available online. To view these files, please visit the journal online (http://dx.doi. org/10.1136/bmjopen-2018028236).

Received 27 November 2018 Revised 16 July 2019 Accepted 11 October 2019

Check for updates

(C) Author(s) (or their employer(s)) 2019. Re-use permitted under CC BY-NC. No commercial re-use. See rights and permissions. Published by BMJ.

${ }^{1}$ Department of Psychology, Lund University, Lund, Sweden ${ }^{2}$ Department of Public Health Science, Section of Epidemiology and Public Health Intervention Research, Karolinska Institutet, Stockholm, Sweden

${ }^{3}$ Department of Clinical Neuroscience, Division of Psychiatry, Karolinska Institutet, Stockholm, Sweden

${ }^{4}$ Department of Clinical Science, Lund, Division of Psychiatry, Lund University, Lund, Sweden

Correspondence to

Dr Catharina Strid;

catharina.strid@psy.lu.se

\section{ABSTRACT}

Objectives Mental health problems and hazardous alcohol consumption often co-exist. Hazardous drinking could have a negative impact on different aspects of health and also negatively influence the effect of mental health treatment. The aims of this study were to examine if alcohol consumption patterns changed after treatment for depression and if the changes differed by treatment arm and patient sex.

Methods This study of 540 participants was conducted in a large randomised controlled trial (RCT) that aimed to compare the effect of internet-based cognitive behavioural therapy, physical exercise and treatment as usual on 945 participants with mild-to-moderate depression. Treatment lasted for 12 weeks; alcohol consumption (Alcohol Use Disorder Identification Test (AUDIT)) and depression (Montgomery Åsberg Depression Rating Scale (MADRS)) were assessed at baseline and 12-month follow-up. Changes in alcohol consumption were examined in relation to depression severity, treatment arm and patient sex.

Results The AUDIT distribution for the entire group remained unchanged after treatment for depression. Hazardous drinkers exhibit decreases in AUDIT scores, although they remained hazardous drinkers according to the cut-off scores. Hazardous drinkers experienced similar improvements in symptoms of depression compared with non-hazardous drinkers, and there was no significant relation between changes in AUDIT score and changes in depression. No differences between treatment arm and patient sex were found.

Conclusion The alcohol consumption did not change, despite treatment effects on depression. Patients with depression should be screened for hazardous drinking habits and offered evidence-based treatment for hazardous alcohol use where this is indicated. Trial registration number DRKS00008745.

\section{INTRODUCTION}

Hazardous drinking is a pattern of alcohol consumption associated with a risk of health problems and the development of alcohol use disorders. In a US study involving 20 000 individuals, Dawson and colleagues ide ${ }^{1}$ ntified several health conditions that may be negatively affected by hazardous alcohol
Strengths and limitations of this study

- The study population was large, despite the high proportions of missing Alcohol Use Disorder Identification Test (AUDIT) data at 12 months.

- No differences between AUDIT 12-month responders and non-responders on baseline measures of age, alcohol consumption, quality of life or level of depression were found, which strengthens the results.

- Since primary alcohol use disorder was an exclusion criterion, findings cannot be generalised to depressed alcohol use disorder populations.

- As this was a secondary analysis of a randomised controlled trial, the study was not sufficiently powered to perform subgroups analyses, which somewhat limits the conclusions draw from the results.

consumption, including liver diseases, nicotine dependence, illicit drug use and psychosocial problems. Despite this, the assessment of alcohol habits in routine healthcare is often neglected. ${ }^{2}{ }^{3}$ As mental health and substance use problems frequently co-exist, assessing alcohol habits in persons affected with mental health problems is important.

Hazardous drinking is usually defined by the number of drinks consumed during a typical week or on a single occasion (binge drinking). ${ }^{4} \mathrm{~A}$ commonly used measure in both healthcare and clinical research is the Alcohol Use Disorder Identification Test (AUDIT), which has shown to have good psychometric properties in several studies. ${ }^{5-7}$ AUDIT has also been used in general population studies. $^{8}$

The lifetime prevalence of depression in women is estimated to be around $20 \%$, which is double that of men. ${ }^{910}$ Hazardous drinking and alcohol use disorder, on the contrary, are more common in men. ${ }^{4}$ Compared with the general population, those affected by 
depression more frequently report patterns of hazardous alcohol consumption-this applies for both sexes-although men more often report these drinking patterns. ${ }^{11}$ The causal association between alcohol use and depression is not clear; however, most studies support the idea that heavy drinking increase the risk for future depression rather than vice versa. ${ }^{12}$ Studies have also shown that hazardous drinking can negatively impact the treatment of depression and anxiety symptoms. Gajecki and colleagues ${ }^{13}$ found that among patients receiving internetbased cognitive behaviour therapy (ICBT) $(n=1601)$ hazardous drinking negatively affected the treatment outcomes in panic disorder. Similarly, in a study of psychiatric outpatients, Bahorik and colleagues ${ }^{14}$ found over a 6-month period that regular hazardous drinking led to slower reductions in depressive and anxiety symptoms. Since hazardous drinking could have a negative impact on several health outcomes, it is important to determine if and how hazardous drinking patterns change following depression treatment.

Participants in the current study were part of a large randomised controlled multicenter study aiming to compare three different treatments for depression; ICBT, physical exercise (PE) and treatment as usual (TAU). The objectives were to increase access to evidence-based and effective treatments for patients with mental health problems in primary care. Previous analyses showed that, compared with TAU, both ICBT and PE were associated with significantly larger improvements. ${ }^{15-17}$

In a secondary analysis of these data, we found that hazardous drinking at baseline negatively influenced the treatment outcome of stress, that is, improvements in stress were smaller among hazardous drinkers compared with non-hazardous. ${ }^{18}$

Although common mental health problems and hazardous drinking frequently co-exist, it remains unclear whether hazardous drinking patterns change following treatment for depression. The aim of this study was to examine if depression treatment was associated with changes in alcohol consumption. A secondary aim was to determine if the changes in consumption differed between treatment arms (ICBT, PE and TAU) and between patient sex.

\section{METHODS}

\section{Study design}

A more detailed description of the full randomised controlled trial (RCT) study design is presented in earlier work. ${ }^{16}{ }^{17}$ Briefly, the study was conducted on patients with mild-to-moderate depression recruited through primary care units in six different regions in Sweden during 2011-2014. Exclusion criteria were severe somatic illness, a primary alcohol or drug use disorder or severe psychiatric problems requiring psychiatric care. Patients judged by clinicians to fulfil the inclusion criteria were screened with the Patient Health Questionnaire-9 (PHQ-9) assessing symptoms of depression and those who obtained $\geq 9$ points were invited to participate. PHQ-9 is a commonly used and easily administered measure for assessing depression. ${ }^{19}$ At baseline, a battery of questionnaires were completed and the patients were randomly allocated by an independent external organisation to one of three treatment arms, ICBT, PE or TAU. Treatment lasted for 12 weeks and follow-ups were made at 3 months (post-treatment) and 12 months (end-point). Alcohol consumption was assessed at baseline and at 12 months only. To reach as many participants as possible, several of the 12-month follow-ups were conducted in a shorter format by phone, which did not include AUDIT. The present study is a secondary analysis of this RCT.

\section{Patient and public involvement}

Patients were not involved in the study design or in the conduct of the study. Representatives from the primary healthcare sector were involved in both the design and the implementation of the study results. Laymen reports from the RCT study are publicly available in Swedish.

\section{Participants}

In total, 945 participants were included in the RCT. The majority was women $(73 \%)$ and the mean age was 43 (SD 12) years. At baseline, 937 participants completed the Montgomery Åsberg Depression Rating Scale (MADRS), assessing depression severity, ${ }^{20}$ and the AUDIT assessing alcohol use. At 12-month follow-up, 797 of the participants completed the MADRS, and 540 of them also answered the AUDIT. The high proportion of missing 12-month follow-up AUDIT data was mostly due to the short telephone assessment where the AUDIT was not included. No differences at baseline were found between AUDIT 12-month responders, $\mathrm{n}=540$, and non-responders, $\mathrm{n}=405$, on age, alcohol consumption, severity of depression and health-related quality of life.

When comparing AUDIT 12-month responders with non-responders, differences were found on depression severity at 12 -month follow-up $(n=797)$. The AUDIT nonresponders $(n=264)$ scored higher on MADRS 12 months compared with responders $(\mathrm{n}=533), t(797)=-2.4, \mathrm{p}=0.02$. However, none of the groups scored in the mild to moderate depressed range (average scores) based on the chosen cut-off scores, $M$ and ( $S D), 10.1$ (7.7), respectively, 11.6 (8.7). The Consolidated Standards of Reporting Trials (CONSORT) diagram (figure 1) shows the flow through the study.

Since no problematic differences between AUDIT 12-month responders and non-responders were found, the results in this study are based on the participants who responded to the AUDIT at 12 months, $\mathrm{n}=540$ (539 responded to the AUDIT both at baseline and 12 months).

\section{Measures}

The pattern of alcohol use was measured by AUDIT, which consists of 10 items scoring both consumption and alcohol-related problems occurring during the last 


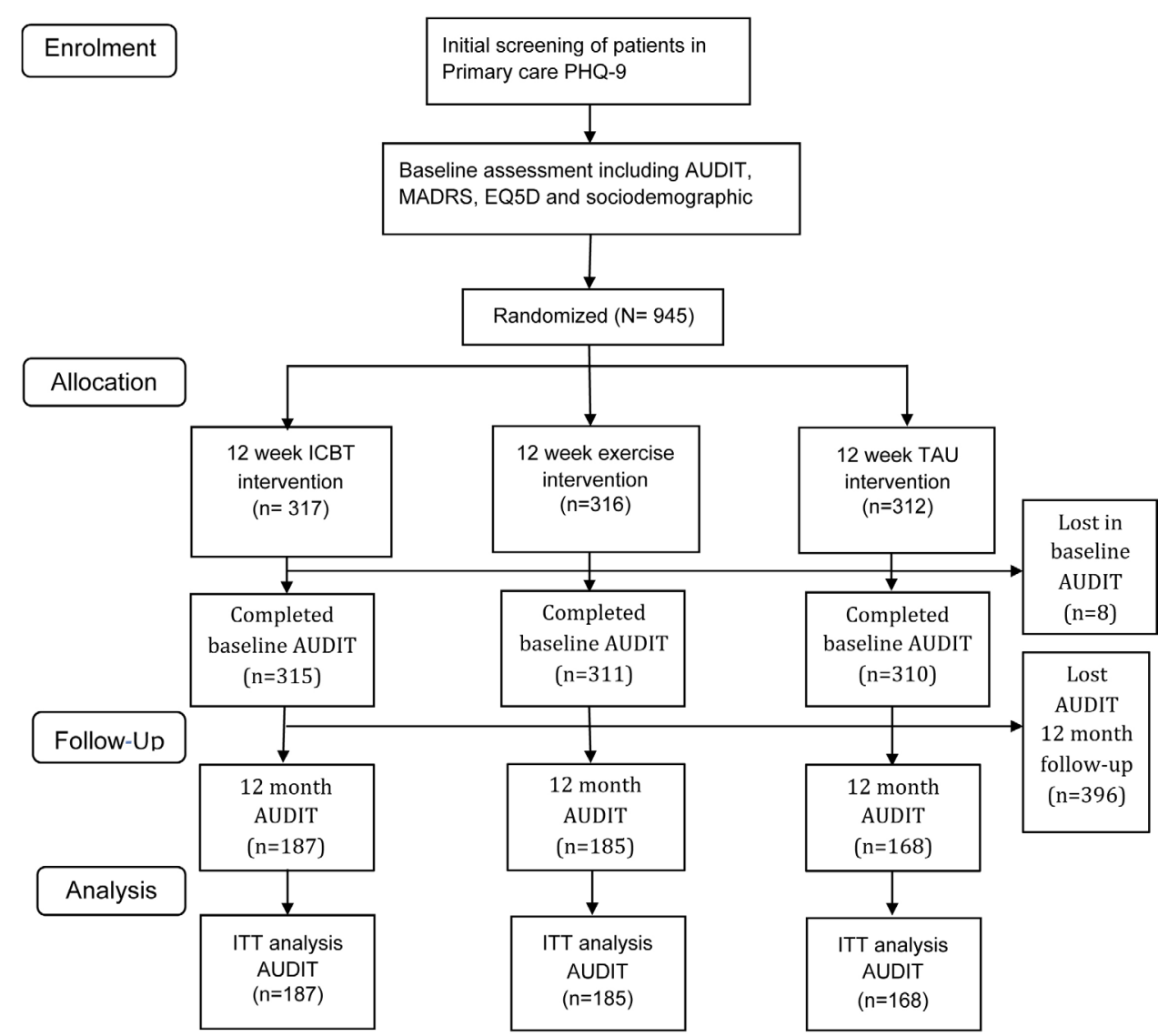

Figure 1 The Consolidated Standards of Reporting Trials (CONSORT) diagram shows the participants' flow through the study. AUDIT, Alcohol Use Disorder Identification Test; EQ5D, EuroQol-5D-3L; ICBT, internet-based cognitive behaviour therapy; ITT, intention to treat; MADRS, Montgomery Åsberg Depression Rating Scale; PHQ-9, Patient Health Questionnaire; TAU, treatment as usual.

year. The first three items AUDIT-C, measure alcohol consumption, both the amount of glass in a week and at one occasion. Items 4-10 measure problems related to alcohol consumption. Results are based on both the total scale, the short consumption scale and the problem drinking scale. The recommended cut-off scores used to identify hazardous drinkers were $>8$ for men and 6 for women. ${ }^{7}$ The instrument has shown acceptable validity and reliability in primary care and is frequently used both in clinical settings and in research. ${ }^{4}$

Depression was assessed with the MADRS; a wellknown clinician-rated questionnaire that has shown good reliability and validity. ${ }^{20}{ }^{21}$ It consists of 10 items rating sadness, anxiety, sleep, appetite, concentration, initiative, ability to engage, and negative and suicidal thoughts. Each item is scored 0-6 (total scores range from 0 to 60 ) and higher scores indicate more severe depression. Several studies have been conducted to define the optimal cutoff scores on the MADRS severity and these vary slightly depending on the population surveyed ${ }^{22-24}$ In this study, we have used Snaith's (1986) recommended cut-off scores for moderate depression (20) and for severe (35), but a higher cut-off for mild depression (12), which is often used by clinicians. ${ }^{25}$
Health-related quality of life was assessed using the EuroQol-5D-3L (EQ-5D), a patient-reported outcome measure developed by EuroQol European Organisation. ${ }^{26}$ The EQ-5D consists of five items with three response alternatives, capturing five health domains; mobility, personal care, daily activities, pain and anxiety/depression. The scores used are based on an algorithm for societal preferences and total scores range from -0.59 to $1.0{ }^{27}$ The mean score in the general population is approximately $0.8 .^{28}$

Education level data was merged into two subgroups: (a) primary and secondary school and (b) 2 years postsecondary or tertiary education. Employment data was merged into four subgroups: (a) employed/studying, (b) disability pension/old age pension, (c) unemployed and (d) sick leave. Civil state data was merged into (a) living together and (b) living alone.

\section{Treatments}

The ICBT programme was delivered by the Internet Psychiatry Unit in Stockholm, Sweden. It was based on an individually tailored programme for depression originally developed by Johansson and colleagues, ${ }^{29}$ and then modified to fit the outcomes in the REGASSA study. The 
treatment was 12 weeks long and included 33 modules based on psychological interventions for problems such as worry, insomnia or pain, but no module focused the reduction of alcohol consumption. The received modules were chosen to address the specific problems presented by the patient in measures conducted before treatment start. The first three modules and the last one were however mandatory and consisted of psychoeducation of depression, behavioural activation and relapse prevention, all standard CBT interventions for depression. The treatment included therapist support consisting of written messages. Symptoms of depression were continuously monitored, and patients with suicidal behaviour were contacted by a clinician for additional help.

The average number of accessed modules was 7.8 (SD 5.1) compared with an expected number of 12-13 modules for full adherence to the programme.

$\mathrm{PE}$ was conducted at independently run fitness centres (Friskis \& Svettis) outside the healthcare, with several locations across Sweden. The programme consisted of three $60 \mathrm{~min}$ exercise sessions and one meeting with a trainer or physiotherapist per week for 12 weeks. Within the exercise arm, participants were randomly allocated to three different conditions; light exercise (ie, Pilates and yoga), moderate aerobic exercise and vigorous aerobic exercise. Exercise intensity was objectively assessed using heart rate monitors worn during the exercise classes. Before and after treatment, the participants completed a bicycle submaximal fitness test.

Participants attended an average of 14.5 one-hour classes out of 36 possible, which corresponds to approximately $40 \%$ of the recommended classes.

TAU was received in primary care and included, on average, one to two visits to the General practitioner (GP), which was similar to the number of GP visits participants in ICBT and PE received during the treatment. ${ }^{30}$ Approximately $30 \%-40 \%$ of the participants in TAU received some psychosocial treatment compared with $10 \%-20 \%$ in the ICBT and PE groups. Around one-third of the participants used antidepressants during the study period, which was similar in TAU compared with ICBT and PE. On average, they had 6.6 visits $(S D$ 7.4). Almost one-fourth of the participants in TAU reported receiving no treatment at all.

\section{Statistical analysis}

In the statistical analyses, both parametric and nonparametric tests were applied. To make sure there were no selection biases, baseline differences between the study sample, $n=540$, and the non-responders, $n=405$, were compared on age, alcohol consumption, depression severity and health-related quality of life using independent sample t-tests. $\chi^{2}$ tests assessed baseline differences between the study sample and the non-responders on education level, gender, treatment group, hazardous drinkers, alcohol-related problems and depression severity. Comparisons on baseline variables between hazardous and non-hazardous drinkers were examined with the same methods.

Paired sample $t$-tests were used to compare differences in alcohol consumption and depression between baseline and 12-month follow-up. Due to multiple comparisons, the Sidak equation for corrected $p$ values was used and the $p$ value was set to $<0.004$. The McNemar test was used to analyse the changes in proportions of hazardous drinkers at baseline and at 12 months. Since the correlations between AUDIT and MADRS and between AUDIT and age were low and not significant, we used a mixed analysis of variance (ANOVA) to calculate differences in alcohol consumption between patient sex; two groups (male vs female) $\times$ two time (AUDIT baseline and 12 months), and between treatment arms; three groups (CBT, PE and TAU) $\times$ two time (AUDIT baseline and 12 months). Time was designed as a within-subject variable. The calculations were performed without covariates in the model and with MADRS and age entered as covariates to make sure that these variables did not affect the associations with alcohol. The relationship between the change in depression and the change in alcohol consumption was measured in a linear regression model.

\section{RESULTS}

\section{Baseline characteristics}

The participants' baseline data are presented in table 1 . More than $80 \%$ were mildly to moderately depressed and $21 \%$ had a hazardous drinking pattern. The healthrelated quality of life, 0.55 , was lower than the general population which is estimated to be around $0.8 .^{28}$

When comparing hazardous $(\mathrm{n}=115)$ with nonhazardous $(n=424)$ drinkers at baseline, there were no differences in severity of depression or health-related quality of life. Compared with non-hazardous drinkers, a significantly higher proportion of hazardous drinkers were male $\chi^{2}=6.94, \mathrm{p}=0.008$, and more hazardous drinkers were living alone $\chi^{2}=8.8, p=0.003$. Hazardous drinkers were also significantly younger $t=2.77$ (537), $\mathrm{p}=0.006$ (table 1).

\section{Differences between baseline and 12-month follow-up}

There were no within-group differences between baseline and 12 months in AUDIT total scores, the AUDIT problem scale or in AUDIT-C consumption scores. This was true for the total sample and for males and females separately (table 2 ).

Depression reduced significantly with an average score change of $M(S D), 11.2$ (9.1), $\mathrm{p}<0.001$, Cohen's $d=1.54$. Hazardous drinking did not differ by sex; all groups significantly reduced their depression scores (table 2 ).

The $\chi^{2}$ test showed significant differences in proportions of hazardous and non-hazardous drinkers at baseline and 12 months for the total group $\chi^{2}=178.02, \mathrm{p}<0.001$, males $\chi^{2}=54.08, \mathrm{p}<0.001$ and females $\chi^{2}=113.31, \mathrm{p}<0.001$ (table 2). The differences were further examined with the McNemar test, which showed no significant change 
Table 1 Descriptive data and comparisons between hazardous and non-hazardous drinkers at baseline $n=539$

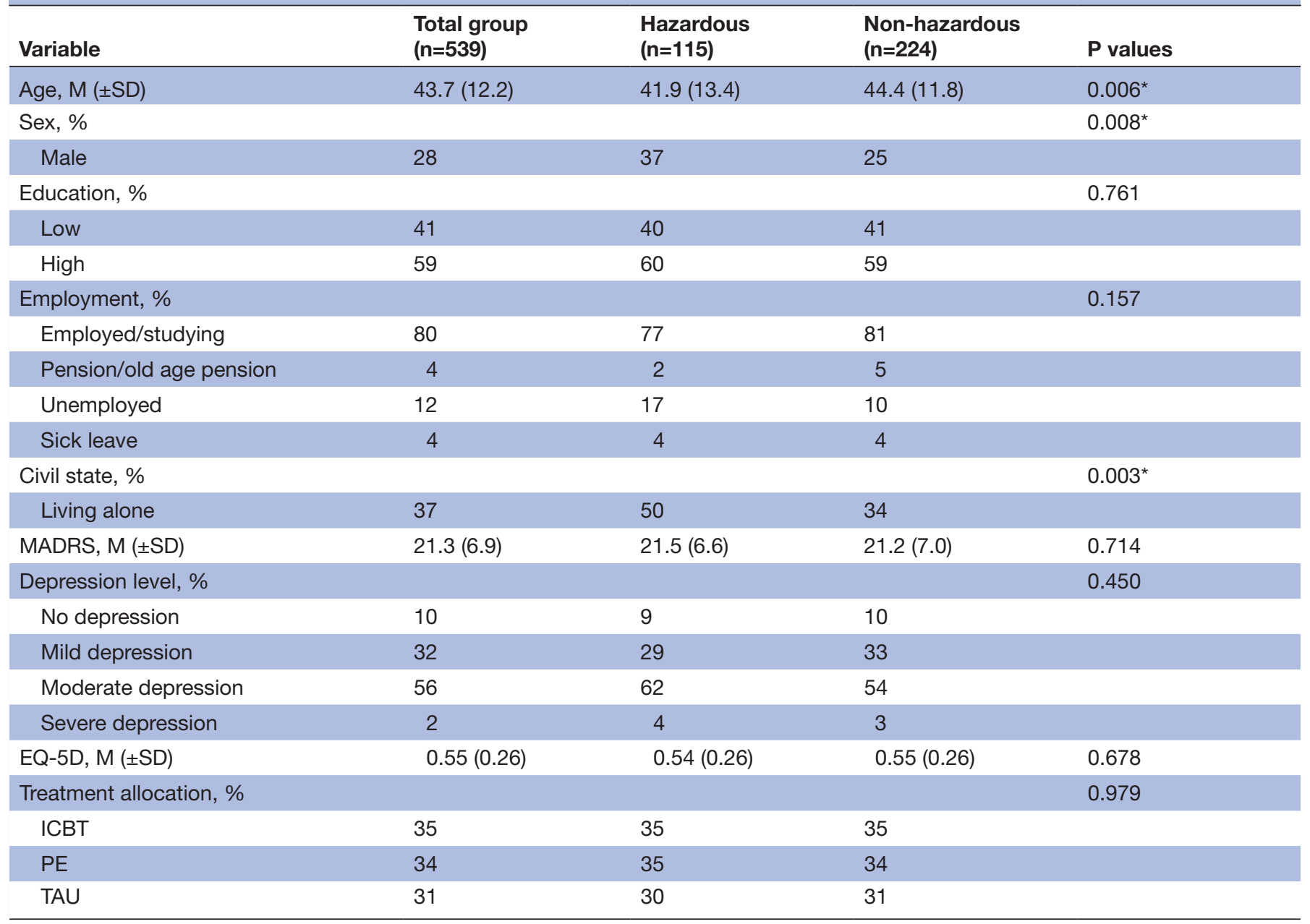

*Significant at $p$ level $<0.01$

EQ-5D, EuroQuality of Life 5 Dimensions; ICBT, internet-based cognitive behaviour therapy; MADRS, Montgomery Åsberg Depression Rating Scale; PE, physical exercise; TAU, treatment as usual.

from non-hazardous to hazardous drinking (or vice versa) between baseline and 12 months. The number of people who changed from hazardous to non-hazardous was about equal to the proportion who changed in the opposite direction (table 3).

The ANOVA test on AUDIT total scores showed, from baseline to 12 months, no significant group differences between treatment arms $F=2.92$ (1, 536), p=0.088 or between patient sex $F=3.78$ (1, 537), p=0.053 and no significant interaction effects between treatment arms and time $F=0.63(2,537), \mathrm{p}=0.531$ or patient sex and time $F=0.80(1,537), \mathrm{p}=0.37$. The results remained nonsignificant when controlling for age and depression. However, the ANOVA test showed differences on AUDIT scores between hazardous and non-hazardous drinkers, in that hazardous drinkers significantly decreased their AUDIT total scores over time $F=35.8$ (1, 537), p<0.001. The interaction effects between time and consumption group were significant $F=61.5$ (1, 537), p<0.001, indicating that hazardous drinkers decreased their scores, while non-hazardous drinkers slightly increased their scores. Although hazardous drinkers decreased their AUDIT scores from $M=10.1$ ( $S D$ 3.6) to $M=8.3$ ( $S D$ 4.6), they remained hazardous drinkers according to the cutoff scores. The results are shown in figure 2 .

The change in AUDIT was not significantly related to the change in MADRS, and this was true for the total group, for hazardous and non-hazardous drinkers and for the treatment arms separately. The change in AUDIT explained $0.3 \%-1.9 \%$ of the total variance of the change in MADRS.

\section{DISCUSSION}

This is one of the first studies to examine the effects of depression treatment on alcohol consumption. In the total sample, depression severity reduced significantly between baseline and 12-month follow-up, and the within-group treatment effects were large, both among hazardous and non-hazardous drinkers. However, the main study finding was that the alcohol consumption did not change over time despite clinically meaningful 
Table 2 Differences, $M( \pm S D)$, in AUDIT total score, AUDIT problem scale, AUDIT-C and MADRS total score between baseline and 12-month follow-up

\begin{tabular}{lcccc}
\hline Variables & Baseline & $\mathbf{1 2}$ & Diff & P value \\
\hline AUDIT total $(n=539)$ & $4.3(3.8)$ & $4.1(3.7)$ & $0.2(2.7)$ & 0.085 \\
\hline Male $(n=149)$ & $5.8(4.3)$ & $5.4(4.2)$ & $0.4(2.7)$ & 0.092 \\
\hline Female $(n=390)$ & $3.7(3.4)$ & $3.5(3.3)$ & $0.1(2.7)$ & 0.324 \\
AUDIT problem scale $(n=536)$ & $1.3(2.5)$ & $1.1(2.3)$ & $0.2(2.0)$ & 0.014 \\
\hline Male $(n=149)$ & $2.1(3.0)$ & $1.7(2.6)$ & $0.4(0.4)$ & 0.049 \\
\hline Female $(n=387)$ & $1.0(2.2)$ & $0.9(2.1)$ & $0.1(1.9)$ & 0.104 \\
\hline AUDIT-C $(n=536)$ & $2.9(1.9)$ & $2.9(1.9)$ & $0.0(1.5)$ & 0.906 \\
Male $(n=149)$ & $3.7(2.1)$ & $3.7(2.1)$ & $0.0(1.4)$ & 0.865 \\
\hline Female $(n=387)$ & $2.6(1.7)$ & $2.7(1.8)$ & $0.1(1.5)$ & 0.810 \\
MADRS $(n=524)$ & $21.3(6.9)$ & $10.0(7.7)$ & $11.2(9.1)$ & $<0.001^{*}$ \\
Male $(n=144)$ & $21.5(6.5)$ & $10.6(8.4)$ & $10.9(9.2)$ & $<0.001^{*}$ \\
Female $(n=380)$ & $21.2(7.1)$ & $9.8(7.5)$ & $11.3(9.0)$ & $<0.001^{*}$ \\
\hline Hazardous drinkers $(n=112)$ & $21.4(6.6)$ & $9.5(7.2)$ & $11.9(8.5)$ & $<0.001^{*}$ \\
Non-hazardous drinkers, $(n=411)$ & $21.2(7.0)$ & $10.2(7.9)$ & $11.1(9.2)$ & $<0.001^{*}$ \\
Hazardous drinkers, \% & 21 & 23 & 2 & $<0.001^{*}$ \\
\hline Male & 29 & 37 & 8 & $<0.001^{*}$ \\
\hline Female & 19 & 17 & 2 & $<0.001$ \\
\hline
\end{tabular}

*Significant at $\mathrm{p}<0.004$.

AUDIT, Alcohol Use Disorder Identification Test; AUDIT-C, Alcohol Use Disorder Identification Test-Consumption; MADRS, Montgomery Åsberg Depression Rating Scale.

treatment effects on depression. A pattern was observed in which hazardous drinkers' AUDIT scores decreased, while non-hazardous drinkers' scores slightly increased. However, these results could reflect regression to the mean rather than meaningful changes after treatment. The proportion of hazardous drinkers remained the same at 12 months follow-up.

Table 3 Changes in number of hazardous and nonhazardous drinkers from baseline to 12-month follow-up for total group $n=540$, male $n=149$ and female $n=391$

\begin{tabular}{lccc}
\hline Baseline & $\begin{array}{l}\text { Non-hazardous } \\
\mathbf{1 2} \text { months }\end{array}$ & $\begin{array}{l}\text { Hazardous } \\
\mathbf{1 2} \text { months }\end{array}$ & P value \\
\hline All & 381 & 43 & 0.428 \\
$\begin{array}{l}\text { Non- } \\
\text { hazardous } \\
\text { Hazardous }\end{array}$ & 35 & 80 & \\
$\begin{array}{l}\text { Male } \\
\begin{array}{l}\text { Non- } \\
\text { hazardous }\end{array}\end{array}$ & 87 & 19 & 0.031 \\
$\begin{array}{l}\text { Hazardous } \\
\text { Female }\end{array}$ & 7 & 36 & \\
$\begin{array}{l}\text { Non- } \\
\text { hazardous }\end{array}$ & 294 & 24 & 0.677 \\
Hazardous & 28 & 44 & \\
\hline
\end{tabular}

These results have implications for clinical practice. As noted, depression and hazardous drinking frequently co-exist, which means that clinicians are often faced with complex health problems that require treatment. ${ }^{31-33} \mathrm{In}$

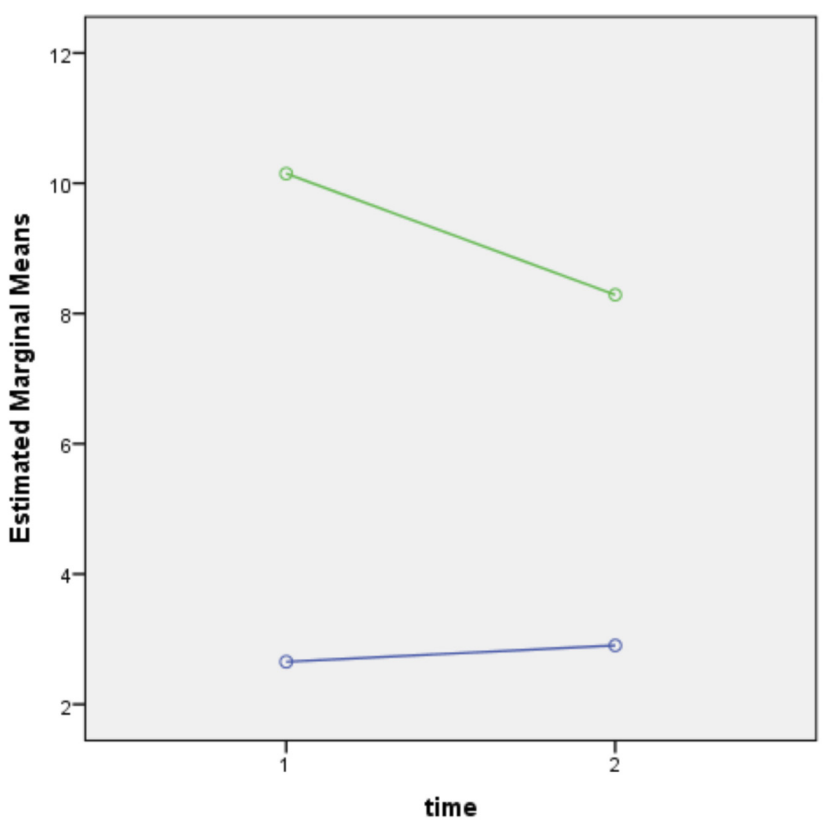

Figure 2 The graph shows the AUDIT scores from baseline to 12 months for hazardous (green) and non-hazardous (blue) drinkers. AUDIT, Alcohol Use Disorder Identification Test. 
this context, it is useful to know whether depression treatment also influences alcohol use, especially among highrisk consumers. Our findings add the observation that even if treatments are effective for depression, hazardous drinking may remain unchanged, along with the risk of developing an alcohol use disorder. Research on treatments with a focus on both alcohol use and depression has confirmed the need to provide interventions, which address hazardous drinking for patients with co-occurring problems. ${ }^{34}{ }^{35}$ However, a recently published review showed that the evidence for combined treatment is limited. ${ }^{36}$ Future studies are needed comparing the effects of alcohol treatment versus combined alcohol and depression treatment, among patients with co-occurring depression and hazardous drinking. Although women are more often affected by depression, hazardous drinking and alcohol-related problems are more common in men, thus male patients should get extra attention about their alcohol use.

If treatment for depression does not have any impact on hazardous drinking, and those with depression exhibit a higher prevalence of problematic drinking compared with the general population, ${ }^{11}$ then it is important to screen alcohol consumption habits in those presenting with depression. Indeed, screening for alcohol and providing brief interventions have demonstrated effectiveness in reducing alcohol use. ${ }^{237-39}$ However, asking patients about their alcohol use and offer interventions for hazardous drinking is seldom performed in primary care, ${ }^{23} 40$ and time constraints are common reasons. Finding ways to implement screening routines for alcohol use is of importance.

The results showed that there were treatment effects on depression for both hazardous and non-hazardous drinkers. Earlier results, on the contrary, have shown that participants with hazardous drinking at baseline were more stressed during and after treatment, and it seems that they had less treatment effect on stress compared with non-hazardous drinkers. ${ }^{18}$

This study showed that the hazardous drinkers did not change their drinking patterns after treatment, which adds strength to earlier results on hazardous drinking and stress. Hazardous drinkers became less depressed after treatment; however, their perceived stress level decreased less compared with non-hazardous drinkers. This might be attributed to the fact that their drinking behaviour remained largely unchanged.

In sum, patients with mental health problems should, as part of routine care, be asked about their alcohol use. Furthermore, those who report hazardous drinking should receive short interventions to help reduce their drinking, since hazardous alcohol use can negatively influence treatment effects on anxiety, symptom of anxiety and depression and stress. ${ }^{13} 1418$ Treatments focusing on mental health problems alone appear to have little effect on hazardous drinking. Combined interventions for reducing alcohol consumption could potentially be administered as part of the assessment.

\section{Strengths and limitations}

More than $40 \%$ of the 12-month follow-ups were conducted by phone, and a shorter assessment was used, which did not include the AUDIT. This would explain the large proportion of missing AUDIT data at 12 months. Even if the missing AUDIT data was high, the study population was large and the AUDIT data robust. At baseline compared with the responders, those with no 12-month AUDIT data did not differ on depression level, quality of life or sociodemographic data, which adds strengths to the results. Some limitations are acknowledged. This was a secondary analysis of an RCT and not a prospectively designed RCT. Thus, the analyses may not have been sufficiently powered to detect small but clinically relevant effects or to perform subgroups analyses, which somewhat limits the conclusions draw. Alcohol use disorder as primary diagnosis was an exclusion criteria for the study; therefore, findings should not be generalised to depressed alcohol use disorder populations. In the PE group, the adherence was low (40\%); higher rates may have reduced consumption further.

\section{CONCLUSION}

The positive treatment effects on depression did not lead to changes in alcohol consumption. The treatments were targeted towards depression and, to the best of our knowledge, no interventions for hazardous drinking were delivered. This suggests that treatments for depression alone are not enough to change hazardous alcohol consumption, which is shown to be higher than average in depressed populations. ${ }^{11}$ To screen for and offer evidence-based interventions for hazardous consumption in patients with mental health problems are important. Research on how to effectively implement interventions addressing hazardous drinking in depressed patients is needed. Further research investigating the effects of interventions for hazardous use without alcohol use disorder in patients with depressive disorders is also needed.

Acknowledgements For helpful statistical advice, we thank late Peter Höglund.

Contributors CS was involved in the design, the acquisition of data, made major contributions to the statistical analysis and interpretation of data, and drafting the manuscript. MH prepared the data set, was involved in data interpretation and writing the manuscript. YF was the principal investigator of the REGASSA study, responsible for the design and data acquisition and has contributed to critical revision of the manuscript. MK was involved in the acquisition of data and has contributed to critical revision of the manuscript. AÖ was the vice principal investigator of the REGASSA study, involved in the design, the statistical analyses and interpretation of the data and in writing the manuscript. All authors have read and approved the manuscript.

Funding The REGASSA study was funded by REHSAM through Vårdal Foundation and Stockholm, Kronoberg, Västmanland and Blekinge county councils, Skåne and Västra Götaland regional councils in Sweden.

Disclaimer These funding sources had no part in study design, data analysis or writing the manuscript.

Competing interests None declared.

Patient consent for publication Not required.

Ethics approval The REGASSA study was approved by the regional ethical review board at Karolinska Institutet in Stockholm (Dnr 2010/1779-31/4) and 
retrospectively registered in German clinical trials DRKS00008745. Before allocation, a written informed consent was obtained from each patient

Provenance and peer review Not commissioned; externally peer reviewed.

Data availability statement Data may be obtained from a third party and are not publicly available.

Open access This is an open access article distributed in accordance with the Creative Commons Attribution Non Commercial (CC BY-NC 4.0) license, which permits others to distribute, remix, adapt, build upon this work non-commercially, and license their derivative works on different terms, provided the original work is properly cited, appropriate credit is given, any changes made indicated, and the use is non-commercial. See: http://creativecommons.org/licenses/by-nc/4.0/.

\section{REFERENCES}

1 Dawson DA, Li T-K, Grant BF. A prospective study of risk drinking: at risk for what? Drug Alcohol Depend 2008;95:62-72.

2 Holmqvist M, Bendtsen P, Spak F, et al. Asking patients about their drinking. A national survey among primary health care physicians and nurses in Sweden. Addict Behav 2008;33:301-14.

3 Satre DD, Leibowitz AS, Mertens JR, et al. Advising depression patients to reduce alcohol and drug use: factors associated with provider intervention in outpatient psychiatry. Am J Addict 2014;23:570-5.

4 Bradley KA, DeBenedetti AF, Volk RJ, et al. AUDIT-C as a brief screen for alcohol misuse in primary care. Alcohol Clin Exp Res 2007;31:1208-17.

5 Saunders JB, Aasland OG, Babor TF, et al. Development of the Alcohol Use Disorders Identification Test (AUDIT): WHO Collaborative Project on Early Detection of Persons with Harmful Alcohol Consumption-II. Addiction 1993;88:791-804.

6 Babor TF, Higgins-Biddle JC, Saunders JB, et al. Audit the alcohol use disorders identification test: guidelines for use in primary care. 2nd edn. World Health Organization, Department of Mental Health and Substance Dependence, 2001. http://www.who.int/substance abuse/publications/audit/en/

7 Bergman $\mathrm{H}$, Källmén $\mathrm{H}$. Alcohol use among SWEDES and a psychometric evaluation of the alcohol use disorders identification test. Alcohol Alcohol 2002;37:245-51.

8 Lundin A, Hallgren M, Balliu N, et al. The Use of Alcohol Use Disorders Identification Test (AUDIT) in Detecting Alcohol Use Disorder and Risk Drinking in the General Population: Validation of AUDIT Using Schedules for Clinical Assessment in Neuropsychiatry. Alcohol Clin Exp Res 2015;39:158-65.

9 Kessler RC, Berglund P, Demler O, et al. The epidemiology of major depressive disorder: results from the National comorbidity survey replication (NCS-R). JAMA 2003;289:3095-105.

10 Roca M, Gili M, Garcia-Garcia M, et al. Prevalence and comorbidity of common mental disorders in primary care. J Affect Disord 2009;119:52-8

11 Åhlin J, Hallgren M, Öjehagen A, et al. Adults with mild to moderate depression exhibit more alcohol related problems compared to the general adult population: a cross sectional study. BMC Public Health 2015;15:542.

12 Boden JM, Fergusson DM. Alcohol and depression. Addiction 2011;106:906-14.

13 Gajecki M, Berman AH, Sinadinovic K, et al. Effects of baseline problematic alcohol and drug use on Internet-based cognitive behavioral therapy outcomes for depression, panic disorder and social anxiety disorder. PLoS One 2014;9:e104615:1-10.

14 Bahorik AL, Leibowitz A, Sterling SA, et al. The role of hazardous drinking reductions in predicting depression and anxiety symptom improvement among psychiatry patients: a longitudinal study. $J$ Affect Disord 2016;206:169-73.

15 Hallgren M, Kraepelien M, Öjehagen A, et al. Physical exercise and Internet-based cognitive-behavioural therapy in the treatment of depression: randomised controlled trial. $\mathrm{Br} J$ Psychiatry 2015;207:227-34.

16 Hallgren M, Helgadóttir B, Herring MP, et al. Exercise and Internetbased cognitive-behavioural therapy for depression: multicentre randomised controlled trial with 12-month follow-up. Br J Psychiatry 2016;209:414-20.

17 Strid C, Andersson C, Forsell Y, et al. Internet-based cognitive behaviour therapy and physical exercise - effects studied by automated telephone assessments in mental ill-health patients; a randomized controlled trial. Br J Clin Psychol 2016;55:414-28.

18 Strid C, Andersson C, Öjehagen A. The influence of hazardous drinking on psychological functioning, stress and sleep during and after treatment in patients with mental health problems: a secondary analysis of a randomised controlled intervention study. BMJ Open 2018;8:e019128.

19 Kroenke K, Spitzer RL, Williams JB. The PHQ-9: validity of a brief depression severity measure. J Gen Intern Med 2001;16:606-13.

20 Montgomery SA, Ásberg M. A new depression scale designed to be sensitive to change. Br J Psychiatry 1979;134:382-9.

21 Quilty LC, Robinson JJ, Rolland J-P, et al. The structure of the Montgomery-Åsberg depression rating scale over the course of treatment for depression. Int J Methods Psychiatr Res 2013:22:175-84.

22 Snaith RP, Harrop FM, Newby DA, et al. Grade scores of the Montgomery-Åsberg depression and the clinical anxiety scales. $\mathrm{Br}$ J Psychiatry 1986;148:599-601.

23 Hawley CJ, Gale TM, Sivakumaran T, et al. Defining remission by cut off score on the MADRS: selecting the optimal value. J Affect Disord 2002;72:177-84.

24 Müller MJ, Himmerich H, Kienzle B, et al. Differentiating moderate and severe depression using the Montgomery-Asberg depression rating scale (MADRS). J Affect Disord 2003;77:255-60.

25 Montgomery SA, Huusom AKT, Bothmer J. A randomised study comparing escitalopram with venlafaxine $\mathrm{XR}$ in primary care patients with major depressive disorder. Neuropsychobiology 2004;50:57-64.

26 EuroQol. Available: www.euroqol.org [Accessed 12 Sep 2012].

27 Dolan P. Modeling valuations for EuroQol health states. Med Care 1997;35:1095-108.

28 Burström K, Johannesson M, Diderichsen F. Health-related quality of life by disease and socio-economic group in the general population in Sweden. Health Policy 2001;55:51-69.

29 Johansson R, Sjöberg E, Sjögren M, et al. Tailored vs. standardized Internet-based cognitive behavior therapy for depression and comorbid symptoms: a randomized controlled trial. PLoS One 2012;7:e36905.

30 Kraepelien M, Mattsson S, Hedman-Lagerlöf E, et al. CostEffectiveness of Internet-based cognitive-behavioural therapy and physical exercise for depression. BJPsych Open 2018;4:265-73.

31 Sullivan LE, Fiellin DA, O'Connor PG. The prevalence and impact of alcohol problems in major depression: a systematic review. Am J Med 2005;118:330-41

32 Baker AL, Kavanagh DJ, Kay-Lambkin FJ, et al. Randomized controlled trial of MICBT for co-existing alcohol misuse and depression: outcomes to 36-months. J Subst Abuse Treat 2014:46:281-90.

33 McBride O, Cheng HG, Slade T, et al. The role of specific alcohol-related problems in predicting depressive experiences in a cross-sectional national household survey. Alcohol Alcohol 2016;51:655-63.

34 Riper H, Andersson G, Hunter SB, et al. Treatment of comorbid alcohol use disorders and depression with cognitive-behavioural therapy and motivational interviewing: a meta-analysis. Addiction 2014;109:394-406.

35 Baker AL, Thornton LK, Hiles S, et al. Psychological interventions for alcohol misuse among people with co-occurring depression or anxiety disorders: a systematic review. J Affect Disord 2012;139:217-29.

36 Hobden B, Bryant J, Carey M, et al. Finding the optimal treatment model: a systematic review of treatment for co-occurring alcohol misuse and depression. Aust N Z J Psychiatry 2018;52:737-50.

37 Kaner EFS, Beyer FR, Muirhead C, et al. Effectiveness of brief alcohol interventions in primary care populations (review). Cochrane Database System Rev 2018:CD004148.

38 Eberhard S, Nordström G, Höglund P, et al. Secondary prevention of hazardous alcohol consumption in psychiatric out-patients: a randomised controlled study. Soc Psychiatry Psychiatr Epidemiol 2009;44:1013-21.

39 Álvarez-Bueno C, Rodríguez-Martín B, García-Ortiz L, et al. Effectiveness of brief interventions in primary health care settings to decrease alcohol consumption by adult non-dependent drinkers: a systematic review of systematic reviews. Prev Med 2015;76(Suppl):S33-8

40 Foulds J, Wells JE, Lacey C, et al. Harmful drinking and talking about alcohol in primary care: new Zealand population survey findings. Acta Psychiatr Scand 2012;126:434-9. 